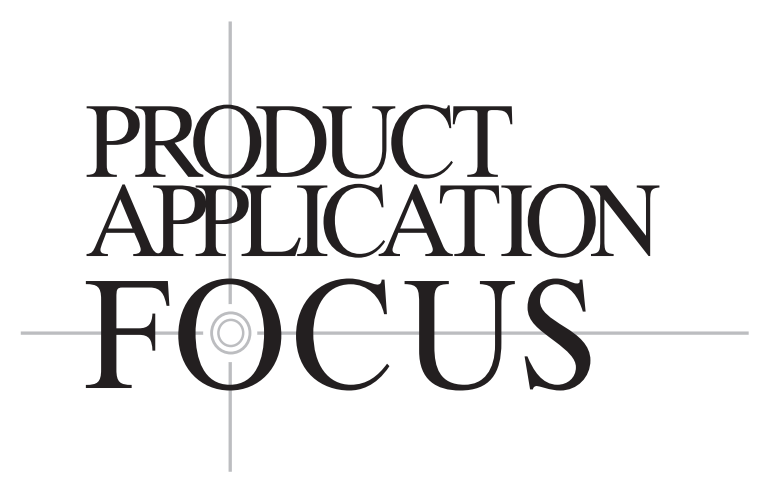

\title{
Cutaneous Wound Analysis Using Hyperspectral Imaging
}

\author{
S.A. Shah ${ }^{1}$, N. Bachrach ${ }^{2}$, S.J. Spear ${ }^{1}$, D.S. Letbetter ${ }^{1}$, R.A. Stone ${ }^{1}$, R. Dhir ${ }^{3}$, \\ J.W. Prichard ${ }^{4}$, H.G. Brown ${ }^{4}$, and W.A. LaFramboise ${ }^{1,4}$ \\ ${ }^{1}$ TissueInformatics, Pittsburgh, PA, ${ }^{2}$ Organogenesis, Canton, MA, ${ }^{3}$ University of \\ Pittsburgh Medical Center, and ${ }^{4}$ Allegheny General Hospital, Pittsburgh, PA, USA
}

BioTechniques 34:408-413 (February 2003)

\begin{abstract}
A correlative bright-field and hyperspectral analysis of fullthickness, cutaneous wounds in a porcine model was undertaken to investigate the efficacy of hyperspectral imaging as an alternate method for wound identification. Analysis of a randomly selected specimen yielded distinct spectral signatures for cutaneous regions of interest including the epidermis, injured dermis, and normal dermis. The scanning of the entire specimen group using these hyperspectral signatures revealed an exclusionary, pseudo-color pattern whereby a central wound region was consistently defined by a unique spectral signature. An algorithm was derived as an objective tool for the comparison of the wound regions defined by the hyperspectral classification versus the pathologists' manual tracings. The dimensions of the wound identified in the hyperspectral assay did not differ significantly from the wound region identified by the pathologists using standard bright-field microscopy. These data indicate that hyperspectral analysis may provide a high-throughput alternative for wound estimation that approximates standard brightfield imaging and pathologist evaluation.
\end{abstract}

\section{INTRODUCTION}

Current methods for characterizing cutaneous biopsy specimens rely predominantly on standard histochemical techniques to accentuate the diagnostic tissue features necessary for an informed evaluation $(1,3,11)$. However, multifarious monoclonal antibodies and nucleotide probes are now capable of exacting previously unavailable detail from biopsies utilizing immunohistochemical and in situ hybridization procedures. Advances in magnetic resonance, confocal imaging, and optical coherence tomography have provided unique methods to analyze tissues at the cellular and molecular lev- el. A drawback to this specialization has been an upsurge in the costs, time requirements, and complexity of these techniques, leading to their availability only within a restricted purview of academic or commercial laboratories with the finances and technical expertise to support the use of these systems. The rationale underlying the current study is to maximize the information available from conventional histochemical methods for skin tissue through the use of hyperspectral imaging, a technique that incorporates instrumentation common to bright-field imaging. The use of alternate imaging modalities to optimize the analysis of a sample prepared according to a standard histology protocol would eliminate added specimen preparation requirements and provide quantitative data on the wound-healing process. Furthermore, this technique may be automated for rapid screening and diagnosis, thereby expediting the development of wound-healing therapies.

The hypothesis of the current study is that hyperspectral imaging can accelerate and refine the evaluation of cutaneous tissue within currently accepted standards of pathological assessment. Hyperspectral imaging techniques are applied in this context, as a novel optical method by which spatially resolved, spectroscopic analyses are applied to a microscopic tissue sample prepared for standard, bright-field analysis. The process allows for the resolution of detail previously unavailable through bright-field microscopy. While human color vision interprets light content as a function of red, green, and blue components, hyperspectral image classification expands the information available from the conventional observation of histological specimens by resolving hundreds of wavelengths within the visible spectra as well as infrared and ultraviolet regions $(7,10)$. The intensity of emitted, reflected, transmitted, or absorbed light measured in time or frequency domains during hyperspectral imaging is presented as inde- 
pendent data that can be selectively reviewed and interpreted by the operator or attending pathologist.

The focus of our approach is to utilize hyperspectral imaging and analysis techniques to aid the pathologist in delineating wounded versus normal regions of cutaneous tissue sections stained according to a standard hematoxylin and eosin (H\&E) protocol. Specifically, we describe a novel spectral imaging method based on transmitted light to define normal and damaged areas of a uniform, full-thickness wound speci-

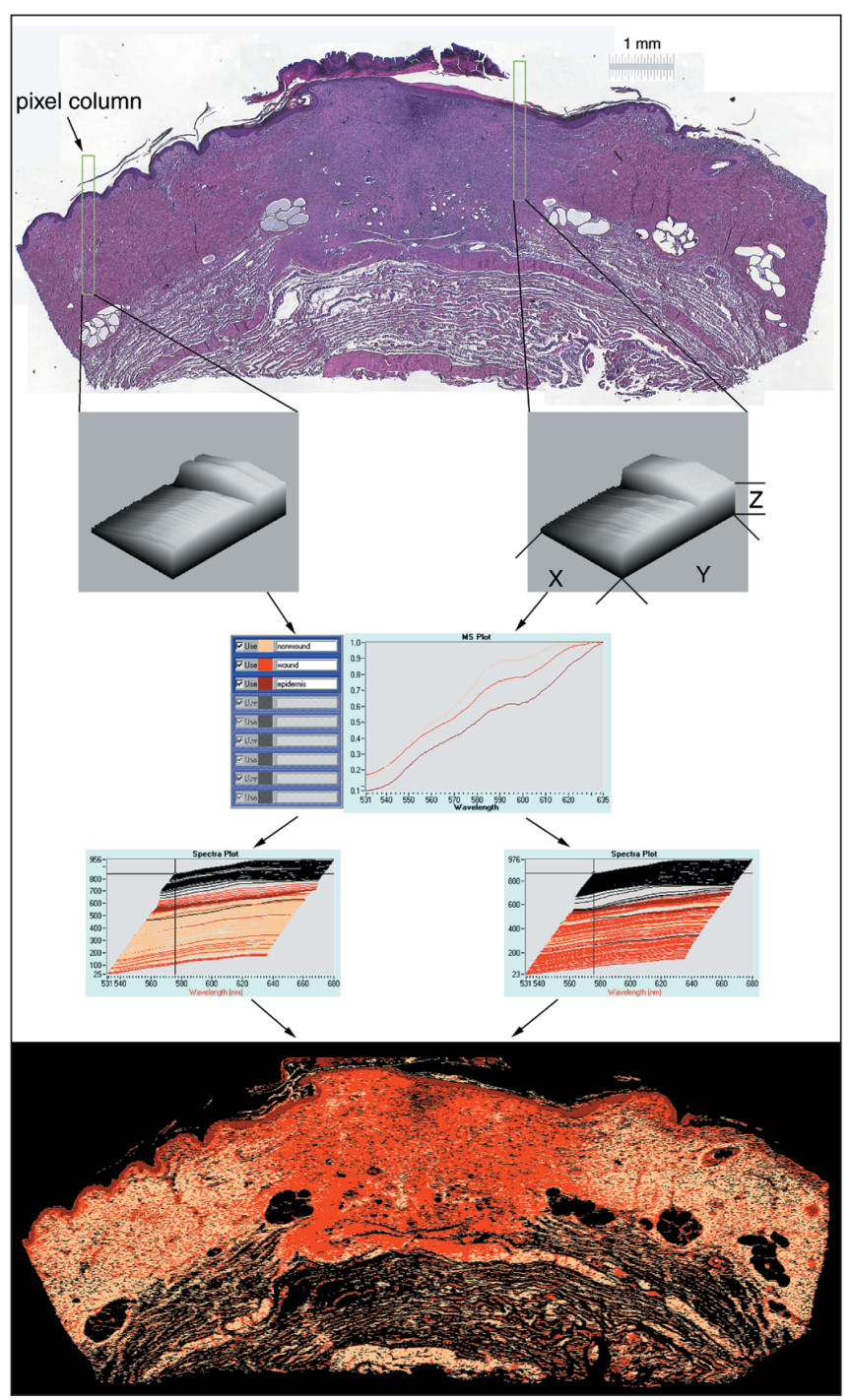

Figure 1. Hyperspectral classification method.

Step 1. Formalin-fixed, paraffin-embedded, cutaneous, porcine tissue with an induced thermal injury was stained with H\&E. Images were under brightfield transmission.

Step 2. 3-D raw spectra of two pixel columns were selected from regions of wound versus normal skin. X, wavelength; Y, pixel position; and Z, intensity.

Step 3. Spectra from the two sampled regions of interest were correlated to obtain a minimal exclusionary spectral library consisting of three master spectra for the classification of epidermis, injured dermis, and normal dermis. The spectra were arbitrarily colored for visual discrimination.

Step 4. Spectral plots for the two pixel columns were classified based on their correlation to the selected spectral library.

Step 5. The entire tissue section of the cutaneous porcine wound was spectrally classified according to the selected spectral library. The correlation values were then applied to each of the specimens in the study, and a series of spectral images was generated for a comparison with the H\&E-stained images. men in a porcine model. The efficacy of this technique as a method of wound identification is demonstrated by direct comparison with standard clinical evaluation.

\section{MATERIALS AND METHODS}

\section{Hyperspectral Image Acquisition}

The hyperspectral imaging system comprised an Olympus ${ }^{\circledR}$ BX40 microscope with a Prior H101 motorized stage (Prior, Cambridge, MA, USA) equipped with a $4 \times$ planar objective [numerical aperture (N.A.) $=0.10$ ] and the PARISS ${ }^{\circledR}$ Imaging Spectrometer (Lightform, Princeton, NJ, USA). Full-thickness cutaneous wound specimens from an Institutional Animal Care and Use Committee-approved porcine model were sectioned and stained (H\&E) on standard microscope slides. The PARISS Imaging Spectrometer was set to capture images with a resolution of $12 \mu \mathrm{m} / \mathrm{pixel}$. Once the exposure and gain settings were established for the spectral camera, the PARISS software allowed the user to define a region of interest (ROI) that characterized the number of pixels and range of wavelengths to be studied. The spectra for each pixel within this ROI were classified against one another (self-classification) by a mathematical correlation algorithm within the PARISS software. The classification is based on a user-defined correlation coefficient ranging from 0.0001 to 1.0000 . Higher correlation settings incorporate more restrictive match criteria for the comparison of spectra. For this study, representative transmission spectra were initially obtained from a randomly selected wound specimen (Figure 1). The spectral results were acquired by the correlation of data from a pixel column of approximately five points for the epidermis (440 $\mu \mathrm{m}^{2}$ ) and 15 points for randomly selected regions of interest within and outside of the dermal wound area $\left(1200 \mu^{2}\right)$. To obtain data representative of the wound area, sampling was performed in a region containing obvious signs of injury visible under bright-field transmission (i.e., a region with breakdown of the cellular epidermal organization plus significant inflammatory cell infiltration). Spectra obtained from each region were automatically translated into a representative, dominant spectrum using the PARISS self-classification algorithm. This process was repeated for each of the three pixel columns. The three classified spectra were compiled into a master spectral library and assigned an arbitrary color. Successive pixel columns of data subtending the entire specimen were acquired and processed through a classification algorithm with a correlation coefficient set to 0.99 , where 1 represents the value at which spectra of scanned pixels must be an exact match to a spectrum in the master library to be classified. The algorithm assigned each pixel the appropriate color that corresponded to the correlative spectra from the master spectral library (Figure 1). Pixels that did not correlate with any of the spectra in the library, including those that represented background, were colored black. This process resulted in a classified image based on an exclusionary hyperspectral criterion that highlighted the epidermis, injured dermis, and normal dermis.

\section{Bright-Field Image Acquisition}

A high-resolution montage of the H\&E-stained specimen analyzed by hyperspectral imaging was generated via the au- 
tomated assembly of contiguous microscope image fields obtained under bright-field transmission at a fixed light intensity and a $4-\mu \mathrm{m} /$ pixel resolution. Images were acquired using a Nikon ${ }^{\circledR}$ E-600 microscope with planar apoepifluorescence $4 \times$ objective (N.A. $=0.13$ ) and a three chip charge-coupled device $(C C D)$ Sony ${ }^{\circledR}$ DXC-950P video camera. The image acquisition process was automated by incorporating a H101A motorized stage and programmed subroutines written in Optimas ${ }^{\circledR} 6.5$ image analysis software (Media Cybernetics, Silver Spring, MD, USA) that allowed for the capture and assembly of contiguous images subtending the entire skin section. To control for CCD variability, light intensity was scaled from 0 (black) to 255 (white) with a programmed white balance. Montage assembly incorporated a flat-field correction for individual image capture to compensate for the heterogeneity of illumination across the image area. All montages were archived in an uncompressed, lossless TIFF format, and analysis processes were performed on a standard PC.

\section{Hyperspectral Image Analysis}

To objectively compare the manual analysis of the brightfield image with the automated hyperspectral classification, an algorithm for edge detection was developed using the Optimas software platform to delineate the borders of the wound area defined by the hyperspectral image. The classified image was divided into contiguous, non-overlapping images of a uniform size that comprised a specific number of pixel clusters. The subroutine employed a color-based threshold to evaluate the density of pixels classified as a wound within each pixel cluster. The entire pixel cluster was assigned as a wound if the density of wound-colored pixels exceeded an arbitrary preset sensitivity value [e.g., if the majority of pixels $(\geq 50 \%)$ in the cluster exhibited the spectral signature of the wound]. To optimize the functionality of the algorithm as a biological index, the dimensions of the pixel cluster and the sensitivity value for edge detection of the wound were tested over a range of sizes and sensitivities. The algorithm performed optimally when adjusted to review $10 \times 10$ pixel units with a sensitivity requirement of $65 \%$ for classification as a wound cluster. Smaller clusters yielded a non-contiguous, punctate wound pattern, while larger pixel clusters extended markedly beyond the wound area circumscribed by the spectral classification.

\section{Bright-Field Image Analysis}

Three accredited, actively practicing surgical pathologists were asked to evaluate the wound dimensions for the selected specimen. Each pathologist was asked to manually and independently "trace the wound border" on the digital image of the H\&E specimen using a standardized drawing program (Adobe ${ }^{\circledR}$ Photoshop ${ }^{\circledR}$ 5.0). The clinicians were provided with the original microscope slide for simultaneous review during the evaluation. Each pathologist was blinded from the assessment of the other two members of the group and the results obtained by hyperspectral classification. The Adobe Photoshop program was used to create an overlay image of the three wound tracings. To establish a standard of reference for the comparison of the hyperspectral results, a maximal wound border was defined by the selection among the pooled tracings in the overlay for the largest wound perimeter.

\section{Comparative Analysis}

The individual pixels classified in the current study represented a biological unit of $12 \mu \mathrm{m}$ on a side. Hyperspectral classification at that level of resolution resulted in pixels interspersed within surrounding normal tissue, with spectral signatures correlating to that of a wound as designated in the master spectral library. To evaluate these images for wound contiguity, it was necessary to assess the hyperspectral content across a larger biological field subtended by multiple pixels. Similarly, the clinical pathologists preferred to make gross observations of wound morphology at a resolution less than the individual pixel, which reflected the fact that many cellular or acellular fibrous structures in these specimens were in a size range exceeding the $12-\mu \mathrm{m}$ pixel dimension.

An edge-detection algorithm based on pixel cluster size and color sensitivity was developed to define a continuous wound perimeter and wound area in the classified hyperspectral image for comparison with the manual tracings. The maximum delineated wound outline from the pathologists' analysis was used as a standard of reference and was superimposed on the classified spectral image with a delineated wound area. The coordinates of the pathologists' reference wound outline and that obtained from the spectral image analysis were calculated and compared to determine the areas of overlap between the two methods of wound delineation. The areas that were identified as a wound by spectral classification that exceeded the area designated by the standard of reference were defined as false positives. False negatives were considered to be those areas outside of the wound region defined by the algorithm for the hyperspectral classification but within the standard of reference defined by the pathologists.

\section{RESULTS}

\section{Hyperspectral Imaging and Analysis}

The hyperspectral classification was performed in advance and separately from the bright-field image analysis. The PARISS algorithm for self-classification yielded a single distinct spectral signature for each of the three regions of interest (epidermis, injured dermis, and normal dermis) when correlation coefficients were set higher than 0.90 . A correlation setting of 0.99 was subsequently employed to compare the entire specimen against the three spectra contained in the master library. The result was an exclusionary spectral pattern whereby a central region of the specimen was defined predominantly by one spectral signature from the master library, distinct from the spectral characteristics of the surrounding tissue (Figure 1). The transformed or arbitrarily colored image based on only these three spectral waveforms appeared to define a contiguous wound region that contrasted with the surrounding normal dermal tissue and a separate epidermal tissue layer. (Figure 1).

Nine additional specimens were subjected to hyperspectral classification using identical settings for light transmission, camera calibration, and correlation coefficient (0.99) as those employed in the analysis of the first specimen. Two biomedical engineers, untrained in medicine or pathology, split the task of performing the imaging. In every case, a centralized region of the biopsy specimen was characterized by a distinct 
spectral data set, compared to surrounding tissue areas and regions near the borders of the section. Rendering the images in arbitrary colors using the spectral library revealed a consistent pattern of a contiguous central core or putative wound region surrounded by tissue of a different spectral classification. Each of the 10 spectral images contained a varying but small percentage of pixels in the normal, uninjured regions whose spectra matched the master spectra isolated from the wound region at the level of correlation selected (0.99). The initial observation suggested that there was a general agreement between the manual tracings of the wound region performed by the pathologist team and those areas delineated by the hyperspectral classification process (Figure 2).

An algorithm was derived as an objective tool for the comparison of the wound regions defined by the hyperspectral classification versus the pathologists' manual tracings. The algorithm sensitivity was affected by $(i)$ the size of the pixel cluster selected for analysis and (ii) the choice of threshold level at which the density of the wound versus normal pixels designated the entire cluster as a wound. The values for these two parameters were varied independently for a single, randomly selected specimen to arrive at optimal settings that were then applied to the remainder of the samples. Preliminary testing indicated that a $10 \times 10$ pixel unit provided a comparator window that approximated the biological field underlying the pathologists' evaluation. Smaller pixel windows produced a spotty or fragmented wound region, while larger windows extended the wound region beyond the limits of the specimen. Similarly, the pixel density threshold was tested over a range from $50 \%$ to $85 \%$ to determine an optimal setting. In every case but one, a setting of $65 \%$ extracted a contiguous wound unit. Higher thresholds yielded fragmented wound areas dispersed throughout the specimen, while lower sensitivities incorporated regions clearly outside the central wound locus. The decision to use $65 \%$ uniformly across the specimens was based on the observation that it minimized both fragmentation and background selection while defining a contiguous wound region.

\section{Comparative Analysis}

The wound area derived by the algorithm for the hyperspectrally classified specimens was quantitatively compared with the pathologists' manual analysis of the bright-field images (Figure 3). The areas overlapped such that, on average, $90 \%$ of the region defined by the algorithm matched with the maximal wound region defined among the three pathologists. The $10 \%$ of the area missed by the algorithm typically fell

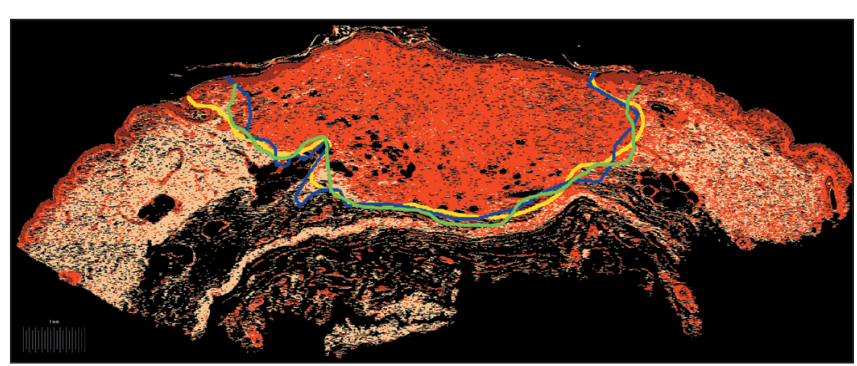

Figure 2. Pathologist versus hyperspectral classification. The wound outlines generated by the pathologists' use of the H\&E-stained specimens were superimposed on the spectrally classified images for a direct comparison with the wound regions demarcated by spectral analysis. within the region selected as a wound by one or two of the pathologists. If their individual assessments were used instead of the reference standard for a maximal wound perimeter, then the area of false negatives was reduced to less than 5\% across the specimens. A complementary situation existed with areas defined as a wound by the algorithm that exceeded the region traced by the pathologists (false positive). These regions averaged $18 \%$ of the size of the manually defined standard of reference. In this case, the exclusion of the reference standard for a maximal wound area resulted in an even larger region defined as a false positive.

\section{DISCUSSION}

The evaluation of cutaneous tissues for the effects of injury and pathology is an arduous, largely manual undertaking that requires rigorous tissue-preparation protocols, expensive processing, optical equipment, and well-trained pathologists for diagnostic assessment. The complexity of this process makes it resistant to automation and a critical bottleneck in the academic and commercial discovery process directed at therapies to expedite the healing of cutaneous wounds associated with injury, surgical resection, pathology, and cosmetic repair. Previous attempts to develop wound imaging and analysis systems for both noninvasive applications and slide specimen analysis have been marginally successful using bright-field techniques and color image processing $(4,5,8)$. However, the demand remains for a system to rapidly and accurately assist in the evaluation of the process of wound repair for use by the pathologist in both clinical and commercial venues.

The results of this investigation indicate that hyperspectral imaging provides an objective means for the classification of cutaneous tissues in full-thickness wounds of porcine specimens. The tool can be used successfully to generate measurements, including wound area and wound perimeter, which are valuable for quantitative wound assessment. These measurements are important as individual parameters in the characterization of wounds and in normalizing data from different wounds (e.g., the number of particular cell types per wound area). Specific advantages are that it requires no unique tissue preparation beyond routine histology protocols and necessitates only minimal training on the part of the system operator.

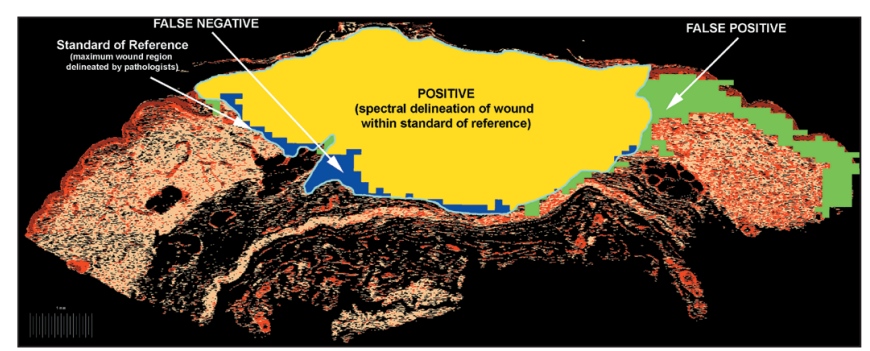

Figure 3. Comparison of manual and automated hyperspectral analysis. The pathologists' wound tracings for each sample were pooled to generate a single standard of reference that represented the maximum delineated wound region. To compare the spectral image to the standard of reference, an edgedetection algorithm based on color thresholding was implemented to highlight the largest contiguous wound region, as defined by the spectral classification for each sample. Areas that are highlighted green illustrate the regions of spectral wound classification that exceed the standard of reference, while those that are highlighted blue represent areas within the standard of reference that are not classified as a wound. The spectral classification of a wound matches that determined by the pathologists in the area highlighted yellow. 
The hardware involved is relatively simple because a typical microscope can be easily adapted to house a hyperspectral imaging system. Only a minimal understanding of pathology is required to calibrate the system for the evaluation of multiple specimens, so it has the potential to operate in a highthroughput, screening environment. While the system will not replace the pathologist, refinement could allow the clinician to rapidly confirm a diagnostic evaluation rather than tediously create it de novo. The development of an algorithm to automate the sampling of areas of a specimen for the isolation of distinctive spectral signatures would remove manual, less objective steps in the current procedure. A similar screening model has proven effective in automated systems for Pap smears and breast cancer; however, those systems require protocols that are more complex than the standard H\&E slide preparation that was adequate for this study (2). An automated hyperspectral system would have the capability of extracting and assessing volumes of data within and outside the visible space of standard sample preparations.

The goal of this investigation was to develop a methodology to utilize transmission-based hyperspectral imaging for the quantification of cutaneous wound areas. The results obtained from the application of the technique over 10 unique samples demonstrated the efficacy of hyperspectral imaging as a system for screening cutaneous wounds. Evaluation of the technique revealed other variables that determined our ability to rigidly test and refine the system. First, the standard of reference, the wound tracings by clinical pathologists, introduced a modicum of variability. Second, the development of a rudimentary algorithm to delineate hyperspectral wound boundaries was necessary to objectively and quantitatively compare the hyperspectral data with the clinical standard of reference. Thus, there was a variability built into the standard of comparison (i.e., the manual tracings against which the algorithm development process must be evaluated). It was important to recognize that the results of this study affirm a role for hyperspectral analysis in wound assessment, particularly when screening for the presence of a wound is critical. The consistency of overlap between the manual tracings and the hyperspectral analysis regarding wound areas and perimeter coordinates suggested that the system may provide considerably more acuity in wound analysis. However, further system refinement will require the development of a consensus criterion for wound definition in the field of dermatopathology.

The results suggest that algorithms can be developed and optimized to more effectively utilize the hyperspectral imaging and analysis process to meet a reference standard. Further development should focus on generating an algorithm to automatically evaluate multiple settings of threshold and window size for each hyperspectral image to outline the optimal contiguous wound region for the classified image. Similarly, the development of overlapping window regions for edge detection could also increase the resolution at which the hyperspectrally classified wound perimeters and areas are delineated.

The experimental paradigm required that the analyses provided by both the pathologists and the hyperspectral procedures focus on defining a wound edge or boundary for gross morphological evaluation. This approach was taken to establish a fundamental basis for the comparison between the manual and automated processes. The assumption that a wound can be delineated by a defined boundary tracing discounts the likelihood that a cellular and biochemical gradient exists at the interface between the wound region and healthy skin. Moreover, efforts to date in the study of wound-healing kinetics have focused on the central wound site as an indicator of healing state (4). Based on the current findings, hyperspectral analysis could be extended to the level of principle component analysis to test for the presence of gradients at the interface between the wound and healthy skin and to provide insight into the healing at the central core of the wound. By simply increasing the magnification and resolution of the microscope, the hyperspectral analysis can be directed specifically at cellular and even subcellular structures. Hyperspectral imaging has demonstrated this ability in other settings, as digital spectra of structures serve as sensitive "fingerprints" that are capable of discriminating morphological abnormalities [e.g., cellular localization of Stat proteins in melanoma precursor lesions (6)]. The results of the current study indicate that hyperspectral analysis performed at these levels could reveal specific changes at the wound interface that contribute to the variability in the clinical evaluation.

\section{REFERENCES}

1.Anderson, R.R. and J.A. Parrish. 1981. The optics of human skin. J. Invest. Dermatol. 77:13-19.

2.Braun, S., N. Harbeck, C. Schindlebeck, P. Shaffer, P. Hutzler, K. Bink, M. Werner, and M. Schmitt. 2001. Using occult metastatic cells in bone marrow and HER2 status for prognostic evaluation of patients with stage I-III breast cancer. American Society of Clinical Oncology, No. 1695.

3.Burkitt, H.G., B. Young, and J.W. Heath. 1993. Skin, p. 153-169. In Functional Histology, Churchill Livingstone, New York.

4.Herbin, M., F.X. Bon, A. Venot, F. Jeanlouis, M.L. Dubertret, L. Dubertret, and G. Strauch. 1993. Assessment of healing kinetics through true color image processing. IEEE Trans. Med. Imaging 12:39-43.

5.Jones, B.F. and P. Plassman. 1995. An instrument to measure the dimensions of skin wounds. IEEE Trans. Biomed. Eng. 42:464-470.

6.Kirkwood, J.M., D.L. Farkas, A. Chakraborty, K.F. Dyer, D.J. Tweardy, J.L. Abernethy, H.D. Edington, S.S. Donnelly, et al. 1999. Systemic interferon- $\alpha($ IFN- $\alpha)$ treatment leads to Stat 3 inactivation in melanoma precursor lesions. Mol. Med. 5:11-20.

7.Levenson, R.M. and D.L. Farkas. 1997. Digital spectral imaging for histopathology and cytopathology. Proc. SPIE 2983:123-135.

8.Mekkes, J.R. and W. Westerhof. 1995. Image processing in the study of wound healing. Clin. Dermatol. 13:401-407.

9.Singer, A.J. and R.A.F. Clark. 1999. Cutaneous Wound Healing. N. Engl. J. Med. 341:738-746.

10.Schultz, R.A., T. Neilsen, J.R. Zavaleta, R. Ruch, R. Wyatt, and H.R. Garner. 2001. Hyperspectral imaging: a novel approach for microscopic analysis. Cytometry 43:239-247.

11.Wilson, B.C. and S.L. Jacques. 1990. Optical reflectance and transmittance of tissues: principles and applications. IEEE J. Quantum Electronics 26:12, 2186-2199.

Address correspondence to Dr. William A. LaFramboise, Department of Cardiothoracic Surgery, Allegheny General Hospital, 320 East North Avenue, Pittsburgh, PA 15212, USA. e-mail: wlaframb@wpahs.org

For reprints of this or any other article, contact Reprints@BioTechniques.com 